\title{
The norm attainment problem for functions of projections
}

\author{
Albrecht Böttcher(i) And Ilya M. Spitkovsky (1)
}

For Bernd Silbermann on his 80th birthday.

\begin{abstract}
The paper is concerned with the problem of identifying the norm attaining operators in the von Neumann algebra generated by two orthogonal projections on a Hilbert space. Every skew projection on that Hilbert space is contained in such an algebra and hence the results of the paper also describe functions of skew projections and their adjoints that attain the norm.
\end{abstract}

Mathematics Subject Classification. Primary 47A30; Secondary 46L89, 47A56, 47B15, 47C15.

Keywords. Two orthogonal projections, Skew projection, Norm attainment.

This note is in the spirit of our paper on Robert Sheckley's answerer for two orthogonal projections [4]. The meta theorem of that paper is that the two projections theorem of Halmos is something like Robert Sheckley's answerer: no question about the $W^{*}$ - and $C^{*}$-algebras generated by two orthogonal projections will go unanswered, provided the question is not foolish. The norm attainment problem asks whether for a given bounded linear operator $A$ there is a unit vector $x$ such that $\|A x\|=\|A\|$. In this generality, a useful answer is not available. Here we pose the question for the case where $A$ is a function of two orthogonal projections or a function of a single skew projection and its adjoint. (Skew projections are frequently also referred to as oblique projections.)

Let $P$ and $Q$ be orthogonal projections acting on a real or complex Hilbert space $\mathcal{H}$. According to Halmos' "Two projections theorem" (see [8] and consult $[3,10]$ for the history and more on the subject), there is a representation of $\mathcal{H}$ as an orthogonal sum

$$
\mathcal{H}=M_{00} \oplus M_{01} \oplus M_{10} \oplus M_{11} \oplus M \oplus M
$$


(the last two summands have the same dimension and are thus identified via an appropriate unitary similarity) with respect to which

$$
P=(1,1,0,0) \oplus\left[\begin{array}{ll}
I & 0 \\
0 & 0
\end{array}\right]
$$

and

$$
Q=(1,0,1,0) \oplus\left[\begin{array}{cc}
I-H & \sqrt{H(I-H)} \\
\sqrt{H(I-H)} & H
\end{array}\right] .
$$

Here and below we use the string $\left(a_{00}, a_{01}, a_{00}, a_{11}\right)$ as an abbreviation for $a_{00} I_{M_{00}} \oplus a_{01} I_{M_{01}} \oplus a_{10} I_{M_{10}} \oplus a_{11} I_{M_{11}}$, while the blocks of the matrix component in $(2),(3)$ are operators on $M$. The selfadjoint operator $H$ has the spectrum $\sigma(H) \subseteq[0,1]$, with 0,1 not being its eigenvalues. In particular, $\min \sigma(H)<1$. Note that $M=\{0\}$ if and only if $P$ and $Q$ commute. The other four subspaces $M_{i j}$ also may or may not be actually present in (1); we will let $\Lambda=\left\{(i, j): \operatorname{dim} M_{i j} \neq 0\right\}$.

According to the Giles-Kummer theorem (see [7] or [3, Theorem 7.1]), the von Neumann algebra $W^{*}(P, Q)$ generated by $P$ and $Q$ consists of the operators $A$ admitting the representation

$$
A=\left(a_{00}, a_{01}, a_{00}, a_{11}\right) \oplus\left[\begin{array}{ll}
\phi_{00}(H) & \phi_{01}(H) \\
\phi_{10}(H) & \phi_{11}(H)
\end{array}\right]
$$

with respect to the decomposition $(1)$ of $\mathcal{H}$. Here $a_{i j}$ are arbitrary complex numbers and $\phi_{i j}$ are (also arbitrary) functions in $L^{\infty}(\sigma(H))$ with respect to the spectral measure of $H$. We will sometimes write the rightmost summand in (4) as $\Phi_{A}(H)$.

The norms of operators from $W^{*}(P, Q)$ were computed in [11, Theorem 10], see also [3, Theorem 7.9]. Namely, for $A$ as in (4),

$$
\|A\|=\max \left\{\max _{(j, k) \in \Lambda}\left|a_{j k}\right|, \max _{x \in \sigma(H)} \sqrt{\frac{\phi(x)+\sqrt{\phi(x)^{2}-4|\omega(x)|^{2}}}{2}}\right\} .
$$

Here $\phi=\sum_{i, j=0,1}\left|\phi_{i j}\right|^{2}$ is the square of the Frobenius norm of $\Phi_{A}$ and $\omega=$ $\operatorname{det} \Phi_{A}=\phi_{00} \phi_{11}-\phi_{01} \phi_{10}$. Note that always $\phi(x)^{2}-4|\omega(x)|^{2} \geq 0$.

The question we are addressing here is: when is $\|A\|$ attained, i.e., when does there exist a unit vector $x \in \mathcal{H}$ such that $\|A x\|=\|A\|$ ? We will call $A$ a norm attaining operator and write $A \in \mathcal{N}$ if this happens to be the case.

For our purposes it is useful to recall that formula (5) was derived in [11] from the fact that

$$
\lambda_{\max }:=\max _{x \in \sigma(H)} \frac{\phi(x)+\sqrt{\phi(x)^{2}-4|\omega(x)|^{2}}}{2}
$$

is the right endpoint of the spectrum $\sigma\left(\Phi_{A^{*} A}(H)\right)$. Since for every operator $X$ acting on $\mathcal{H}$, we have $X \in \mathcal{N}$ if and only if $X^{*} X \in \mathcal{N}$ if and only if the right endpoint of $\sigma\left(X^{*} X\right)$ is its eigenvalue (see [6] and [9]), we just need to figure out when $\lambda_{\max }$ is (or is not) an eigenvalue of $A^{*} A$. 
To this end, recall that for operators in $W^{*}(P, Q)$, the description of their kernels is also known ([3, Theorem 7.5] or [11, Theorem 1]). There is no need to include its exact form here. A consequence which is important for us reads as follows.

Lemma 1. The kernel of $\Phi_{A}(H)$ is non-trivial if and only if the spectral measure of $\{x \in \sigma(H): \omega(x)=0\}$ is non-zero.

When applied to $A-\lambda I$ in place of $A$, Lemma 1 immediately yields the following.

Lemma 2. Let $A$ be given by (4). Then $\lambda \in \mathbb{C}$ is an eigenvalue of $A$ if and only if the spectral measure of the set $\left\{x \in \sigma(H): \lambda^{2}-\operatorname{trace} \Phi_{A}(x) \lambda+\operatorname{det} \Phi_{A}(x)=\right.$ $0\}$ is non-zero.

Applying Lemma 2 to $A^{*} A$ and taking into account that trace $\Phi_{A^{*} A}=\phi$ and $\operatorname{det} \Phi_{A^{*} A}=|\omega|^{2}$, we see that the eigenvalues $\lambda$ of $A^{*} A$ are characterized by the property that the spectral measure of the set $\left\{x \in \sigma(H): \lambda^{2}-\phi(x) \lambda+\right.$ $\left.|\omega(x)|^{2}=0\right\}$ is non-zero. In particular, $\lambda_{\max }$ is an eigenvalue of $A^{*} A$ (and not just a point of its spectrum) if and only if the spectral measure of the set of all $x \in \sigma(H)$ on which the function

$$
\psi(x):=\phi(x)+\sqrt{\phi(x)^{2}-4|\omega(x)|^{2}}
$$

attains its maximum value is non-zero. Denoting this set by $\Sigma(A)$, we arrive at the following conclusion.

Theorem 3. Let $A$ be the operator given by (4). Then $A \in \mathcal{N}$ if and only if either (i) $\max _{(j, k) \in \Lambda}\left|a_{j k}\right| \geq \sqrt{\lambda_{\max }}$ or (ii) $\max _{(j, k) \in \Lambda}\left|a_{j k}\right|<\sqrt{\lambda_{\max }}$ and the spectral measure of $\Sigma(A)$ is non-zero.

Let now $T$ be a skew projection on $\mathcal{H}$. We assume that $T$ is genuinely skew, which is equivalent to the requirement that $\|T\|>1$. Denote by $P=P_{\operatorname{Ran} T}$ the orthogonal projection onto the range of $T$ and by $Q=P_{\operatorname{Ker} T}$ the orthogonal projection onto the kernel of $T$. Afriat [1] (see also [3, Proposition 1.6]) showed that then $\|P Q\|<1$ and

$$
T=(I-P Q)^{-1} P(I-P Q) .
$$

Moreover, in (1) then $M_{00}=M_{11}=\{0\}$, while $M_{01}$ and $M_{10}$ may or may not be present. Omitting the $M_{00}$ and $M_{11}$ terms in (2) and (3), we obtain

$$
I-P Q=(1,1) \oplus\left[\begin{array}{cc}
H & -\sqrt{H(I-H)} \\
0 & I
\end{array}\right],
$$

and since $I-P Q$ is invertible, so also must be $H$. Formula $(7)$ then gives

$$
T=(1,0) \oplus\left[\begin{array}{c}
I-\sqrt{H^{-1}(I-H)} \\
0
\end{array}\right]=(1,0) \oplus\left[\begin{array}{c}
I-\sqrt{H^{-1}-I} \\
0
\end{array}\right] .
$$

Corollary 4. A skew projection attains its norm if and only if $\min \sigma(H)$ is an eigenvalue of the respective operator $H$. 
Proof. Indeed, for $A=T$, we get from (8) that $\phi(x)=x^{-1}, \omega(x)=0$, and hence $\psi(x)=2 x^{-1}$, which is a monotonically decreasing function. It follows that $\Sigma(T)$ is the singleton $\{\min \sigma(H)\}$. Since $\|T\|>1$, part (i) of Theorem 3 is irrelevant and the assertion follows from part (ii) of that theorem.

From (2) and (3) and the equalities $M_{00}=M_{11}=\{0\}$, we infer that

$$
P Q P=(0,0) \oplus\left[\begin{array}{cr}
I-H & 0 \\
0 & 0
\end{array}\right] .
$$

Thus, if $A=T$, we see that the respective operator $H$ in Corollary 4 is

$$
H=(I-P Q P)|\operatorname{Ran} P=I| \operatorname{Ran} T-P_{\operatorname{Ran} T} P_{\operatorname{Ker} T} \mid \operatorname{Ran} T .
$$

We conclude with some examples. The authors of [2] recently proved that a skew projection $T$ is in $\mathcal{N}$ if and only if the selfadjoint operator $T+T^{*}-I$ belongs to $\mathcal{N}$. The operator $T+T^{*}-I$ appeared in [5] and is therefore called the Buckholtz operator in [2]. The following is an extension of this result.

Example 5. Let $T$ be a skew projection. Then the following are equivalent:

(i) $T \in \mathcal{N}$,

(ii) $T+\alpha T^{*}+\beta I \in \mathcal{N}$ for some $\alpha, \beta \in \mathbb{R}$,

(iii) $T+\alpha T^{*}+\beta I \in \mathcal{N}$ for all $\alpha, \beta \in \mathbb{R}$.

Proof. Fix $\alpha, \beta \in \mathbb{R}$. We have to show that $T \in \mathcal{N} \Longleftrightarrow T+\alpha T^{*}+\beta I \in \mathcal{N}$. According to (8),

$$
A:=T+\alpha T^{*}+\beta I=(1+\alpha+\beta, \beta) \oplus\left[\begin{array}{cc}
(1+\alpha+\beta) I & -\sqrt{H^{-1}-I} \\
-\alpha \sqrt{H^{-1}-I} & \beta I
\end{array}\right] .
$$

Abbreviating $1+\alpha+\beta$ to $s$ and $x^{-1}-1$ to $f(x)$, we obtain

$$
\begin{aligned}
\phi(x)= & (1+\alpha+\beta)^{2}+\beta^{2}+\left(1+\alpha^{2}\right)\left(x^{-1}-1\right)=s^{2}+\beta^{2}+\left(1+\alpha^{2}\right) f(x), \\
\omega(x)= & (1+\alpha+\beta) \beta-\alpha\left(x^{-1}-1\right)=s \beta-\alpha f(x), \\
\psi(x)= & s^{2}+\beta^{2}+\left(1+\alpha^{2}\right) f(x) \\
& +\sqrt{\left(s^{2}+\beta^{2}+\left(1+\alpha^{2}\right) f(x)\right)^{2}-4(s \beta-\alpha f(x))^{2}} .
\end{aligned}
$$

The term under the square root equals

$$
\left(s^{2}-\beta^{2}\right)^{2}+2\left[\left(s^{2}+\beta^{2}\right)\left(1+\alpha^{2}\right)+4 s \beta \alpha\right] f(x)+\left(1-\alpha^{2}\right)^{2} f(x)^{2} .
$$

The function $f(x)$ is monotonically decreasing and non-negative. Since $2|s \beta| \leq$ $s^{2}+\beta^{2}$ and $2|\alpha| \leq 1+\alpha^{2}$, we have $\left(s^{2}+\beta^{2}\right)\left(1+\alpha^{2}\right)+4 s \beta \alpha \geq 0$. Consequently, the term under the square root and therefore also $\psi(x)$ are monotonically decreasing, which implies that $\Sigma(A)=\{\min \sigma(H)\}$. Using that $\min \sigma(H)<1$, we get

$$
\begin{aligned}
\lambda_{\max } & \geq \frac{s^{2}+\beta^{2}+\left(1+\alpha^{2}\right) f(\min \sigma(H))+\left|s^{2}-\beta^{2}\right|}{2} \\
& >\frac{s^{2}+\beta^{2}+\left|s^{2}-\beta^{2}\right|}{2}=\max \left(s^{2}, \beta^{2}\right),
\end{aligned}
$$

and Theorem 3(ii) implies that $A \in \mathcal{N}$ if and only if the spectral measure of the singleton $\{\min \sigma(H)\}$ is positive, that is, if and only if $\min \sigma(H)$ is an 
eigenvalue of $H$. This together with Corollary 4 shows that $A \in \mathcal{N} \Longleftrightarrow T \in \mathcal{N}$, as desired.

Example 6. Let $T$ be a skew projection. Put $T^{(2)}=T T^{*}, T^{(3)}=T T^{*} T$, and more generally, $T^{(m)}:=\underbrace{T T^{*} T T^{*} T \ldots}_{m}$. Then, for each $m$,

$$
T^{(m)} \in \mathcal{N} \Longleftrightarrow T \in \mathcal{N} \text {. }
$$

Proof. From (8), we infer that if $m=2 k$ is even, then

$$
T^{(m)}=\left(T T^{*}\right)^{k}=(1,0) \oplus\left[\begin{array}{cc}
H^{-k} & 0 \\
0 & 0
\end{array}\right] .
$$

This implies that $\psi(x)=2 x^{-2 k}$ and thus $\Sigma\left(T^{(m)}\right)=\{\min \sigma(H)\}$. Taking into account that $\min \sigma(H)<1$, we obtain as above from Theorem 3(ii) and Corollary 4 that $T^{(m)} \in \mathcal{N} \Longleftrightarrow T \in \mathcal{N}$. Finally, since $A \in \mathcal{N} \Longleftrightarrow A A^{*} \in \mathcal{N}$, we conclude that

$$
T^{(2 k+1)} \in \mathcal{N} \Longleftrightarrow T^{(4 k+2)} \in \mathcal{N} \Longleftrightarrow T \in \mathcal{N},
$$

which gives the assertion in the case of odd $m$.

Example \%. Let $\left\{\omega_{n}\right\}_{n=1}^{\infty}$ be a sequence of positive real numbers that converge monotonically to zero and let $T$ be the skew projection on $\ell^{2}(\mathbb{N})$ defined by the infinite matrix

$$
T=\operatorname{diag}\left\{\left[\begin{array}{cc}
1 & -\omega_{n} \\
0 & 0
\end{array}\right]_{n=1}^{\infty}\right\} .
$$

Then $T \in \mathcal{N}$. Put $A=T T^{*}+T^{*} T-T-T^{*}-I$. If $\omega_{n}=1 / n$, then $A \notin \mathcal{N}$, but if $\omega_{n}=2 / n$, then $A \in \mathcal{N}$.

Proof. It is clear that $T \in \mathcal{N}:$ the norm is attained at the vector

$$
\left(1 / \sqrt{1+\omega_{1}^{2}},-\omega_{1} / \sqrt{1+\omega_{1}^{2}}, 0,0, \ldots\right)^{\top} \in \ell^{2}(\mathbb{N}) .
$$

To treat the operator $A$, we employ Theorem 3 . We have $M_{i j}=\{0\}$ for all $i, j$ and may therefore write

$$
T=\left[\begin{array}{c}
I-\sqrt{H^{-1}-I} \\
0 \\
0
\end{array}\right] \text { with } H=\operatorname{diag}\left(x_{n}\right)_{n=1}^{\infty},
$$

where $\sqrt{x_{n}^{-1}-1}=\omega_{n}$, that is, $x_{n}=1 /\left(1+\omega_{n}^{2}\right)$. Straightforward computation gives

$$
A=\left[\begin{array}{cc}
H^{-1}-2 I & 0 \\
0 & H^{-1}-2 I
\end{array}\right] .
$$

Thus, $\psi(x)=2\left(2-x^{-1}\right)^{2}$. If $\omega_{n}=1 / n$, then

$$
\sigma(H)=\left\{\frac{n^{2}}{n^{2}+1}: n \in \mathbb{N}\right\} \cup\{1\}=\left\{\frac{1}{2}, \frac{4}{5}, \frac{9}{10}, \ldots\right\} \cup\{1\} .
$$


The function $\psi(x)$ takes its maximum on $\sigma(H)$ at $x=1$, with $\lambda_{\max }=\psi(1) / 2=$ 1. Hence $\Sigma(A)=\{1\}$, and as 1 is not an eigenvalue of $H$, Theorem 3(ii) implies that $A \notin \mathcal{N}$. If $\omega_{n}=2 / n$, we have

$$
\sigma(H)=\left\{\frac{n^{2}}{n^{2}+4}: n \in \mathbb{N}\right\} \cup\{1\}=\left\{\frac{1}{5}, \frac{1}{2}, \frac{9}{13}, \ldots\right\} \cup\{1\} .
$$

This time $\psi(x)$ assumes its maximum at $x=1 / 5$, the value of the maximum being $\lambda_{\max }=\psi(1 / 5) / 2=3.24$. It follows that $\Sigma(A)=\{1 / 5\}$, and since $1 / 5$ is an eigenvalue of $H$, we deduce from Theorem 3 (ii) that $A \in \mathcal{N}$.

The last example can be elaborated to great extent. However, we leave it with Israel M. Gelfand: "Explain this to me on a simple example; the difficult example I will be able to do on my own." (http://www.israelmgelfand.com/ edu_work.html)

Funding Open Access funding enabled and organized by Projekt DEAL.

Open Access. This article is licensed under a Creative Commons Attribution 4.0 International License, which permits use, sharing, adaptation, distribution and reproduction in any medium or format, as long as you give appropriate credit to the original author(s) and the source, provide a link to the Creative Commons licence, and indicate if changes were made. The images or other third party material in this article are included in the article's Creative Commons licence, unless indicated otherwise in a credit line to the material. If material is not included in the article's Creative Commons licence and your intended use is not permitted by statutory regulation or exceeds the permitted use, you will need to obtain permission directly from the copyright holder. To view a copy of this licence, visit http://creativecommons. org/licenses/by/4.0/.

Publisher's Note Springer Nature remains neutral with regard to jurisdictional claims in published maps and institutional affiliations.

\section{References}

[1] Afriat, S.: Orthogonal and oblique projectors and the characteristics of pairs of vector spaces. Math. Proc. Cambridge Philos. Soc. 53, 800-816 (1957)

[2] Bala, N., Dhara, K., Sarkar, J., Sensarma, A.: Idempotent, model, and Toeplitz operators attaining their norms. Linear Algebra Appl. 622, 150-165 (2021)

[3] Böttcher, A., Spitkovsky, I.M.: A gentle guide to the basics of two projections theory. Linear Algebra Appl. 432, 1412-1459 (2010)

[4] Böttcher, A., Spitkovsky, I.M.: Robert Sheckley's answerer for two orthogonal projections. Oper. Theory Adv. Appl. 268, 125-138 (2018)

[5] Buckholtz, D.: Inverting the difference of Hilbert space projections. Amer. Math. Monthly 104, 60-61 (1997)

[6] Carvajal, X., Neves, W.: Operators that achieve the norm. Integral Equations Operator Theory 72, 179-195 (2012)

[7] Giles, R., Kummer, H.: A matrix representation of a pair of projections in a Hilbert space. Canada Math. Bull. 14, 35-44 (1971) 
[8] Halmos, P.: Two subspaces. Trans. Amer. Math. Soc. 144, 381-389 (1969)

[9] Pandey, S., Paulsen, V.: A spectral characterization of $\mathcal{A N}$ operators. J. Aust. Math. Soc. 102, 369-391 (2017)

[10] Roch, S., Santos, P.A., Silbermann, B.: Non-commutative Gelfand Theories. Universitext. Springer, London (2011)

[11] Spitkovsky, I.M.: Once more on algebras generated by two projections. Linear Algebra Appl. 208(209), 377-395 (1994)

\author{
Albrecht Böttcher \\ Fakultät für Mathematik \\ TU Chemnitz 09107 \\ Chemnitz \\ Germany \\ e-mail: aboettch@mathematik.tu-chemnitz.de \\ ILyA M. SpitKovsky \\ Division of Science and Mathematics \\ New York University Abu Dhabi (NYUAD) \\ Saadiyat Island \\ P.O. Box 129188 \\ Abu Dhabi \\ United Arab Emirates \\ e-mail: ims2@nyu.edu; \\ ilya@math.wm.edu; \\ imspitkovsky@gmail.com
}

Received: 12 March 2021

Revised: 22 April 2021

Accepted: 7 May 2021. 